OPEN ACCESS

Edited by:

Jorg Conradt,

Royal Institute of Technology, Sweden

Reviewed by:

Alejandro Linares-Barranco,

Sevilla University, Spain

Terrence C. Stewart,

National Research Council Canada

(NRC-CNRC), Canada

*Correspondence:

Carlo Michaelis

carlo.michaelis@

phys.uni-goettingen.de

Received: 30 July 2020 Accepted: 28 October 2020

Published: 26 November 2020

Citation:

Michaelis C, Lehr AB and Tetzlaff C (2020) Robust Trajectory Generation

for Robotic Control on the

Neuromorphic Research Chip Loihi.

Front. Neurorobot. 14:589532.

doi: 10.3389/fnbot.2020.589532

\section{Robust Trajectory Generation for Robotic Control on the Neuromorphic Research Chip Loihi}

\author{
Carlo Michaelis ${ }^{*}$, Andrew B. Lehr and Christian Tetzlaff \\ Department of Computational Neuroscience, University of Göttingen, Göttingen, Germany
}

Neuromorphic hardware has several promising advantages compared to von Neumann architectures and is highly interesting for robot control. However, despite the high speed and energy efficiency of neuromorphic computing, algorithms utilizing this hardware in control scenarios are still rare. One problem is the transition from fast spiking activity on the hardware, which acts on a timescale of a few milliseconds, to a control-relevant timescale on the order of hundreds of milliseconds. Another problem is the execution of complex trajectories, which requires spiking activity to contain sufficient variability, while at the same time, for reliable performance, network dynamics must be adequately robust against noise. In this study we exploit a recently developed biologically-inspired spiking neural network model, the so-called anisotropic network. We identified and transferred the core principles of the anisotropic network to neuromorphic hardware using Intel's neuromorphic research chip Loihi and validated the system on trajectories from a motor-control task performed by a robot arm. We developed a network architecture including the anisotropic network and a pooling layer which allows fast spike readout from the chip and performs an inherent regularization. With this, we show that the anisotropic network on Loihi reliably encodes sequential patterns of neural activity, each representing a robotic action, and that the patterns allow the generation of multidimensional trajectories on control-relevant timescales. Taken together, our study presents a new algorithm that allows the generation of complex robotic movements as a building block for robotic control using state of the art neuromorphic hardware.

Keywords: robot control, neuromorphic computing, Loihi, anisotropic network, spiking neural network, computational neuroscience

\section{INTRODUCTION}

During infancy, humans acquire fine motor control, allowing flexible interaction with real world objects. For example, most humans can effortlessly grasp a glass of water, despite variations in object shape and surroundings. However, achieving this level of flexibility in artificial autonomous systems is a difficult problem. To accomplish this, such a system must accurately classify inputs and take appropriate actions under noisy conditions. Thus, increasing robustness to input noise is crucial for the development of reliable autonomous systems (Khalastchi et al., 2011; Naseer et al., 2018).

Neuromorphic hardware is based on highly parallel bio-inspired computing, which employs decentralized neuron-like computational units. Instead of the classical separation of processing and memory, on neuromorphic hardware information is both processed and stored in a network of these computational units. Neuromorphic architectures offer faster and more energy-efficient 
computation than traditional CPUs or GPUs (Blouw et al., 2019; Tang et al., 2019), which is a vital feature for autonomous systems. However, porting existing robot control algorithms (e.g., Ijspeert et al., 2002) to neuromorphic hardware is per se ambitious (but see Eliasmith and Anderson, 2004; DeWolf et al., 2016; Voelker and Eliasmith, 2017) and difficult to optimize to the specific hardware architecture. At the same time, the development of new algorithms is also challenging due to the decentralized design principle of neuromorphic hardware as a network of computational units (Lee et al., 2018).

The basic network type for the various neuromorphic architectures developed in recent years (Schemmel et al., 2010; Furber et al., 2014; Davies et al., 2018; Neckar et al., 2018) are spiking neural networks (SNNs), coined third generation neural networks (for review, see Maass, 1997; Tavanaei et al., 2019). In particular, the reservoir computing paradigm, such as echo state networks (Jaeger, 2001, 2007) or liquid state machines (Maass et al., 2002), often serves as an algorithmic basis. In reservoir computing a randomly connected SNN provides a "reservoir" of diverse computations, which can be exploited by training weights from the reservoir units to additional units that constitute timedependent outputs of the system.

The internal dynamics of the reservoir or SNN generally provide a sufficient level of variability such that arbitrary output functions on a control-relevant timescale can be read out. However, the system fails if the input is noisy or perturbations arise while the trajectory is being performed (Maass et al., 2002; Sussillo and Abbott, 2009; Laje and Buonomano, 2013; Hennequin et al., 2014). That is to say, spiking dynamics in SNNs are often unstable, meaning that small changes in the initial conditions result in different spiking patterns (Sompolinsky et al., 1988; Van Vreeswijk and Sompolinsky, 1996; Brunel, 2000; London et al., 2010). Thus, when an output is trained using such a spiking pattern, low levels of noise lead to a deviation of the estimated output from the target output and stable trajectories can only be obtained on a timescale of milliseconds. On the other hand, attractor dynamics provide highly stable, persistent activity (Amit, 1992; Tsodyks, 1999); however, they tend to lack the variability in the spiking dynamics required for complex output learning (Nachstedt and Tetzlaff, 2017). This implies a stabilityvariability trade-off, also denoted as a robustness-flexibility tradeoff (Pehlevan et al., 2018).

A number of approaches have been developed in recent years to stabilize the spiking dynamics of SNNs while retaining sufficient variability for output learning (Laje and Buonomano, 2013; Hennequin et al., 2014; Pehlevan et al., 2018; Vincent-Lamarre et al., 2020). To improve stability, recent approaches used feed-forward structures (Pehlevan et al., 2018) or employed supervised learning rules (Laje and Buonomano, 2013). While feed-forward structures provide stable activity patterns, in general these play out on a very fast timescale (Zheng and Triesch, 2014) or require neural/synaptic adaptation such that activity moves between neuron groups (York and Van Rossum, 2009; Itskov et al., 2011; Murray et al., 2017; Maes et al., 2020). And since for supervised learning all states in the network need to be accessible at each computing unit, these so-called global learning rules are not compatible with most neuromorphic hardware.

Thus, achieving stable activity patterns on a control-relevant timescale in a network architecture and learning regime capable of running on neuromorphic hardware remains an open problem. Necessary criteria are that (1) learning or adaptation mechanisms in the $\mathrm{SNN}$ should be local to individual synapses, or synapses should be static, (2) sequential activity patterns should remain active for hundreds of milliseconds, (3) spike patterns should contain sufficient variability for arbitrary output learning, and (4) the network should possess noiserobust neuronal dynamics. Meeting these criteria is especially difficult for recurrent network structures, like reservoir networks. However, the so-called anisotropic network model appears to be a promising candidate (Spreizer et al., 2019). The model is based on a biologically-inspired rule for forming spatially asymmetric nonplastic connections. Thus, synapses are static, meeting the first criterion, and the timescale of activity sequences is on the order of tens to hundreds of milliseconds, fulfilling the second criterion. However, whether the model also fulfills the third and fourth criteria, sufficient variability and stability under input noise, has not yet been assessed.

In this paper we use the anisotropic network as a building block for a novel algorithm yielding robust robotic control. We implement the network architecture on Kapoho Bay, a neuromorphic hardware system from Intel containing two Loihi chips (Davies et al., 2018), and show that this approach can be used to learn complex trajectories under noisy input conditions on a control-relevant timescale. Furthermore, we demonstrate that this neuromorphic network architecture can not only robustly represent complex trajectories, but even generalize beyond its training experience.

\section{METHODS}

We first describe the architecture of the novel algorithm implemented on the neuromorphic chip Loihi, which supports robust robotic control of movement trajectories. The anisotropic network and its implementation is then explained in detail. Finally analyses methods to evaluate the implementation of the anisotropic network on Loihi, the stability of its network dynamics, and the learning of complex movement trajectories are described.

\subsection{Architecture of the Algorithm for Robotic Control}

The architecture, shown in Figure 1A, was designed to support the storage and execution of stable movement trajectories in realtime. The architecture consists of an input layer, an anisotropic network layer, and a pooling layer, all of which are fully implemented on Loihi. Spike patterns from the anisotropic network or the pooling layer are read out and serve as the basis for training output units.

The basic computational structure for the robotic control algorithm is the anisotropic network. Excitatory and inhibitory neurons are initialized with local, spatially inhomogeneous 


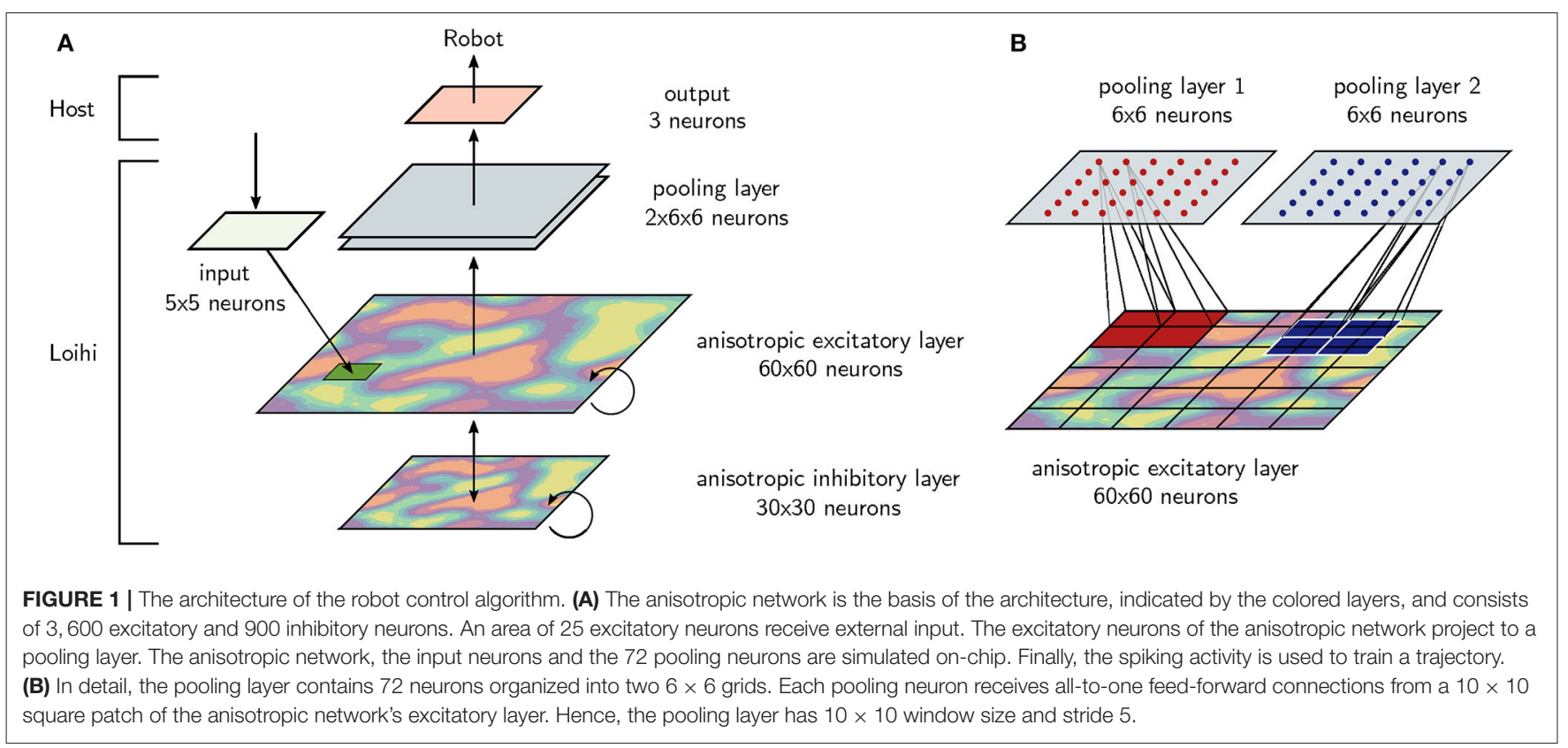

connections as described below. An input is connected to a grid of $5 \times 5$ excitatory neurons in order to start spiking activity with a short input pulse.

The excitatory neurons of the anisotropic network are connected to a pooling layer with 72 excitatory neurons. Pooling layer neurons are organized into two grids with a size of $6 \times 6$ neurons, as shown in Figure 1B. Each neuron in the pooling layer receives input from a $10 \times 10$ group of excitatory neurons from the anisotropic network. These projections are all-to-one and all feed-feedforward weights are equal. In other words, the pooling layer has $10 \times 10$ window size and stride 5 .

Depending on the task, either the excitatory neurons of the anisotropic network or the 72 neurons of the pooling layer are read out. Since reading out data from Loihi is a bottle neck that reduces the simulation speed considerably, the pooling layer is designed to reduce read out and therefore increase simulation speed. Finally, linear regression is applied to the spiking activity of the read-out (see section 2.5).

\subsection{The Anisotropic Network}

We briefly describe the main principles of the anisotropic network. For an in depth treatment, we refer readers to Spreizer et al. (2019).

In locally connected random networks (LCRNs), neurons are distributed in (connectivity) space (e.g., on a $2 \mathrm{D}$ grid or torus) and the connection probability between two neurons decreases (possibly non-monotonically) with the distance between them. Stable bumps of spatially localized activity can arise in LCRNs (Roxin et al., 2005; Hutt, 2008; Spreizer et al., 2017, 2019) and these activity bumps can move through the network in a streamlike manner if spatial asymmetries are introduced into the local connectivity (Spreizer et al., 2019).

The anisotropic EI-network consists of both excitatory and inhibitory neurons arranged on a $2 \mathrm{D}$ torus. Neurons project their axons in a distance dependent way with connection probability decreasing monotonically according to a Gaussian distribution. In a standard LCRN, axon projection profiles are centered at the neuron and axons project symmetrically in all directions. In the anisotropic EI-network, the Gaussian distribution is shifted for excitatory neurons such that connections to other excitatory neurons are formed preferentially in a particular direction (Figure 2A).

A so-called landscape is computed on the torus using Perlin noise (Perlin, 1985), and each point on the grid (neuron) is assigned a direction based on this. The Perlin landscape ensures that the preferred direction of nearby neurons are similar while preferred directions of those far apart are uncorrelated (Figure 2B). Each excitatory neuron's connectivity profile is shifted by one grid point in its preferred direction, resulting in spatially asymmetric but correlated connectivity. When a set of neurons in close proximity are stimulated, spatio-temporal sequences of activity lasting tens to hundreds of milliseconds are elicited (Figure 2C).

Taken together, a biologically plausible rule can generate spatially asymmetric connectivity structures supporting spatiotemporal sequences. Spreizer et al. (2019) show that if (1) individual neurons project a small fraction $(\sim 2-5 \%)$ of their axons preferentially in a specific direction (Figure $2 \mathbf{A}$ ), and (2) neighboring neurons prefer similar directions (Figure 2B), then sequences of neural activity propagate through the network (Figure 2C). This simple generative connectivity rule results in feed-forward paths through the otherwise locally connected random network.

\subsection{Anisotropic Network Implementation}

We adapted the anisotropic EI-network model from Spreizer et al. (2019). Since the total number of connections currently supported by the Loihi NxSDK-API is limited (see section 4), 

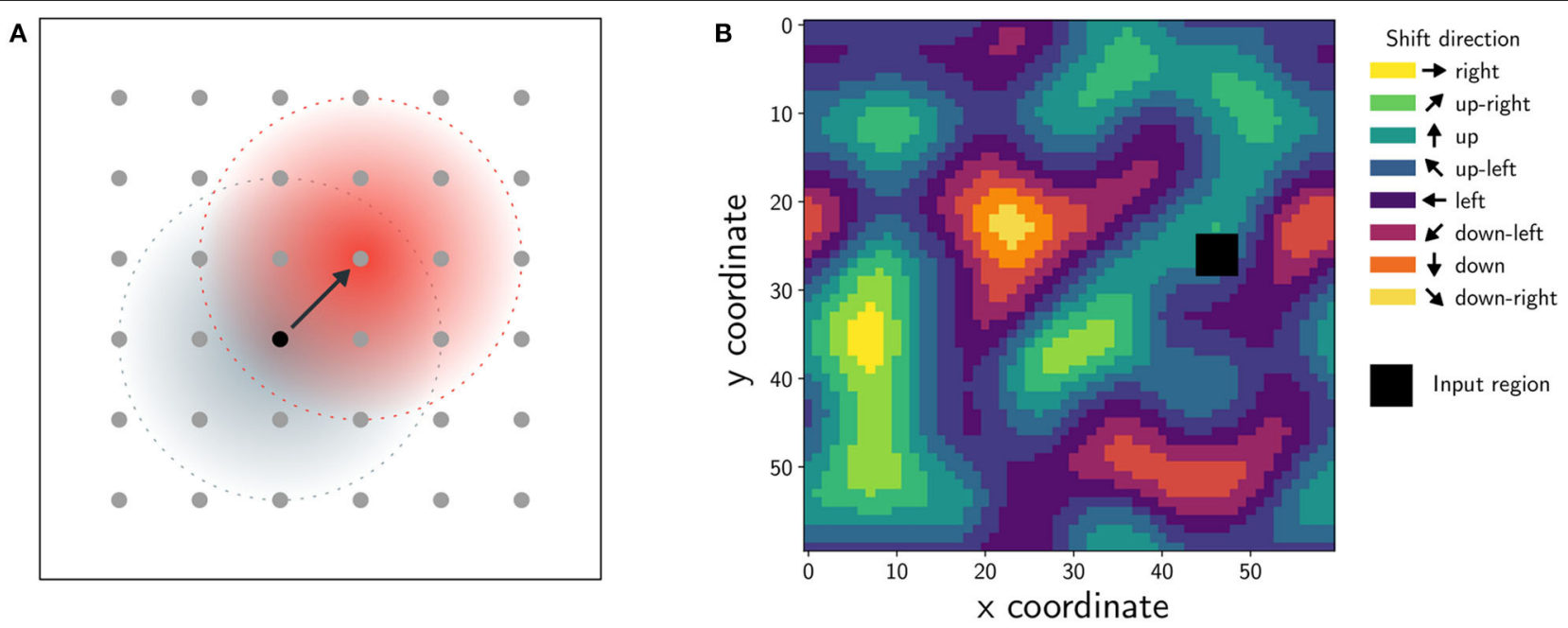

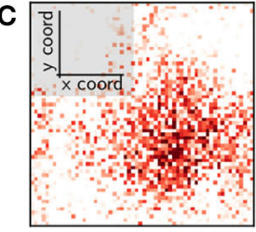

$1-50$

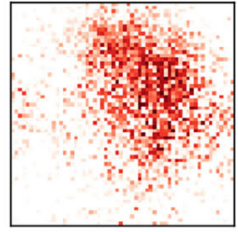

$51-100$

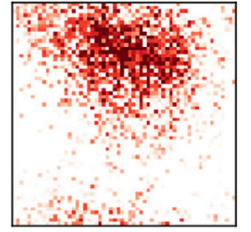

$101-150$

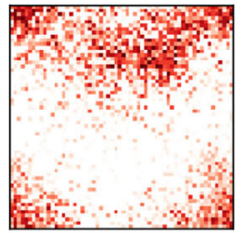

$151-200$

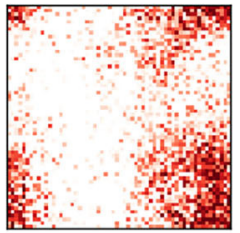

$201-250$

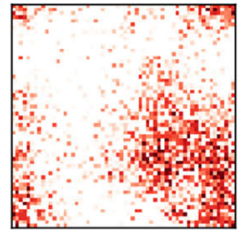

$251-300$

FIGURE 2 | The connectivity underlying the anisotropic network and resulting network dynamics. (A) Neurons are evenly distributed on a $2 \mathrm{D}$ grid, folded to form a torus. The black dot represents a reference neuron. From here outgoing connections can be drawn locally with a Gaussian distribution, either symmetrically (gray-blue) or non-symmetrically (red). In the non-symmetrical case the center of the Gaussian distribution is shifted by one neuron. This shift can be chosen in different ways. Either with the same shift direction for every neuron (homogeneously), a random shift direction for every neuron, or with a specific distribution. (B) If for each neuron the shift direction is chosen based on Perlin noise, the distribution of shift directions for the whole network results in an anisotropic connectivity structure. The black region represents spatially-localized input. (C) Given local input, the anisotropic network forms a bump of activity, which moves in a stream-like pattern through the inhomogeneous structure of connections. Here, we used the Loihi implementation and binned the spikes into non-overlapping time windows. Each graph shows the average firing rate over 50 time steps, color code depicts firing rate.

it was necessary to reduce network size by a factor of four to $n p o p_{E}=3,600$ and $n p o p_{I}=900$. Each neuron projects to $p_{c o n n} \times n p o p_{E}=180$ excitatory targets and $p_{c o n n} \times n p o p_{I}=$ 45 inhibitory targets, where $p_{\text {conn }}=0.05$ is the connection probability. Connection probability decreases with distance according to a Gaussian distribution with space constants given in Supplementary Table 1. We first adapted the anisotropic EInetwork model within NEST and then transferred it to Loihi, tuning the network to qualitatively match the behavior of the NEST simulation.

\section{NEST Implementation}

Neurons were modeled as leaky integrate-and-fire (LIF) neurons, with sub-threshold membrane potential $v$ of neuron $i$ evolving according to:

$$
C_{m} \frac{d v_{i}}{d t}=-g_{L}\left(v_{i}(t)-E_{L}\right)+I_{i}(t)+I_{i}^{i n p u t}(t),
$$

where $C_{m}$ is the membrane capacitance, $g_{L}$ the leak conductance, and $E_{L}$ the reversal potential. For neuron $i, I_{i}(t)$ is the total synaptic current from its recurrent connections and $I_{i}^{\text {input }}(t)$ the current induced by external input.
The total synaptic current $I_{i}(t)$ to neuron $i$ at its recurrent synapses is the sum of the current transients at each of its synapses, $I_{i}(t)=\sum_{j} I_{i j}(t)$. When a pre-synaptic neuron spikes, a current transient is elicited with temporal profile given by an alpha function:

$$
I_{i j}(t)=J^{s y n} \frac{t-t_{j, k}}{\tau_{s y n}} \exp \left(-\frac{t-t_{j, k}}{\tau_{s y n}}\right) .
$$

Note here that the superscript syn can denote both excitatory (exc) and inhibitory (inh) synapses. Synaptic strength is $J^{s y n}$, synaptic time constant is $\tau_{s y n}$, and spike time is $t_{j, k}$ for the $k^{\text {th }}$ spike from neuron $j$.

To compensate for the decreased network size and hence fewer recurrent connections (see above) we scaled up the synaptic weights. The excitatory synaptic current was scaled up by a factor of four to $J^{e x c}=40.0 p A$. To ensure persistent spiking activity in response to an input pulse, the ratio of recurrent inhibition and excitation was reduced to $g=4$. As a result, $J^{i n h}=-g \times J^{e x c}=-160.0 p A$.

Activity was triggered by external input to a subset of neighboring neurons, each of which receives an input pulse of 500 
spikes with synaptic strength $J^{\text {input }}=1.0 p A$ arriving according to a Gaussian distribution with standard deviation of $1 \mathrm{~ms}$.

\section{Loihi Implementation}

For the implementation on neuromorphic hardware we used the research chip Loihi from Intel (Davies et al., 2018), which is a digital and deterministic chip that is based on an asynchronous design. The board we used contains two chips, with each chip providing 128 neuron cores and three embedded x86 CPUs. Each neuron core time-multiplexes the calculation and allows the implementation of up to 1,024 neurons each. We distributed the total of 4,572 utilized neurons (reservoir and pooling layer) with 20 neurons per core. Computation on the chip is performed in discrete time steps and has no relation to physical time. Finally, the Loihi board is connected to a desktop computer, called host in the following, via a serial bus (USB).

We translated the NEST implementation to the NxSDK (version 0.9.5-daily-20191223) for Loihi, provided by Intel labs (Lin et al., 2018). For this, we developed a software framework PeleNet ${ }^{1}$, based on the NxSDK, especially for reservoir networks on Loihi. This framework was used for all simulations in this study.

The Loihi chip implements a leaky integrate-and-fire (LIF) neuron with current-based synapses and the membrane potential $v$ of neuron $i$ evolves according to

$$
\frac{d v_{i}}{d t}=-\tau_{v}^{-1} v_{i}(t)+I_{i}(t)+I_{i}^{i n p u t}(t)-v_{t h} \sigma_{i}(t),
$$

where $\tau_{v}$ describes the time constant, $v_{t h}$ the firing threshold, $I_{i}(t)$ the total synaptic current from recurrent connections, $I_{i}^{\text {input }}(t)$ the current induced by the input, and $\sigma_{i}(t)$ denotes whether neuron $i$ spiked at time $t$. The first term on the right-hand side controls voltage decay, the second/third term increases the voltage according to the synaptic/input currents, and the last term resets the membrane potential after a spike occurs.

While the NEST implementation uses alpha-function shaped synaptic currents (see Equations 1 and 2), Loihi's current-based synapses implement instantaneous rise and exponential decay. The total synaptic current from recurrent connections to neuron $i$ is given by

$$
I_{i}(t)=\sum_{i \neq j} J_{i j}^{s y n}\left(\alpha_{I} * \sigma_{j}\right)(t)+I_{i}^{b i a s},
$$

where $J_{i j}^{s y n}$ is the synaptic strength from neuron $j$ to neuron $i$ which can be excitatory $\left(J^{e x c}\right)$ or inhibitory $\left(J^{i n h}\right)$ and $I_{i}^{\text {bias }}$ is a bias term. The $\sigma_{j}(t)$ represents the incoming spike train from neuron $j$ and $\alpha_{I}(t)$ a synaptic filter. The spike train for a neuron $j$ is given by a sum of Dirac delta functions with

$$
\sigma_{j}(t)=\sum_{k} \delta\left(t-t_{j, k}\right)
$$

\footnotetext{
$\overline{{ }^{1} \text { https://github.com/sagacitysite/pelenet/tree/neurorobotics }}$
}

where $t_{j, k}$ is the time of spike $k$ for neuron $j$. The function simply indicates whether neuron $j$ spiked in time step $t$. The spike train is convolved with a synaptic filter given by

$$
\alpha_{I}(t)=\tau_{I}^{-1} \exp \left(-\frac{t}{\tau_{I}}\right) H(t)
$$

where $\tau_{I}$ is a time constant and $H(t)$ the unit step function.

With Equations (5) and (6), we can bring Equation (4) into a form which is comparable to Equation (2). Setting $I_{\text {bias }}=0$, we get

$$
\begin{aligned}
I_{i}(t) & =\sum_{i \neq j} J_{i j}^{s y n}\left(\alpha_{I} * \sigma_{j}\right)(t) \\
\stackrel{\text { Equation }(5)}{=} & \left.\sum_{i \neq j} J_{i j}^{s y n} \sum_{x} \alpha_{I}(x) \sum_{k} \delta\left((t-x)-t_{j, k}\right)\right) \\
& =\sum_{i \neq j} J_{i j}^{s y n} \sum_{k} \alpha_{I}\left(t-t_{j, k}\right) \\
\stackrel{\text { Equation }(6)}{=} & \sum_{i \neq j} J_{i j}^{s y n} \sum_{k} \tau_{I}^{-1} \exp \left(\frac{t_{j, k}-t}{\tau_{I}}\right) H\left(t-t_{j, k}\right) .
\end{aligned}
$$

Due to the filter, the input current induced by a pre-synaptic spike decays exponentially for each following time step. And instead of rising slowly, at the time of a spike, $t=t_{j, k}$, synaptic current increases by $\tau_{I}^{-1} J_{i j}^{s y n}$. Thus, compared to the neuron model from the anisotropic network implementation in NEST (Spreizer et al., 2019), the hardware-implemented neuron model on Loihi differs since it lacks a current rise time.

\subsection{Comparing the Implementations}

Network activity was started with the input mentioned above and 500 discrete time steps in Loihi and $500 \mathrm{~ms}$ in NEST were recorded. In NEST the resolution was set to $d t=0.1 \mathrm{~ms}$ (see also Supplementary Table 1) per simulation step, while in Loihi a physical time is not defined. After the simulation, the NEST spiking data was binned to $1 \mathrm{~ms}$ to match the Loihi data. Note that, given the refractory period of $2 \mathrm{~ms}$, the binned spike trains still contain binary values, but with a less precise information about the sub-millisecond spike times. In the end, both data sets, the spike trains for NEST and the spike trains for Loihi contained 500 discrete steps.

To compare the spiking patterns between NEST and Loihi quantitatively, we calculated the mean firing rate of groups of excitatory neurons in both networks, which is shown in Figure 3B. For this, we split the two dimensional network topology into a $6 \times 6$ grid, analogous to the grid used for the pooling layer (see Figure 1B) such that each grid position represents a group of 100 neurons. The indices of the groups are chosen from top left to bottom right. For each group, we averaged the firing rate over the 500 time steps resulting in 36 values.

\subsection{Stability and Output Learning}

To analyse the stability (Figure 5) of the network and for the output learning (Figure 6), we applied another protocol. Note 
that from this point on, the NEST implementation was not used. Out of the 25 excitatory neurons connected to the input, we stimulated only 24 neurons such that 1 neuron stays silent (Figure 4A). This input grid then allows 25 different input configurations and therefore 25 different trials with a noise level of $4 \%$. Every trial was recorded for 215 time steps and then the activity was stopped by resetting the membrane voltages. We did this by applying a $\mathrm{C}$ code that runs on one $\mathrm{x} 86$ core on each chip (a so called SNIP). After waiting 30 time steps, the next input was applied to the network. The applied protocol is indicated by arrows in Figure 5A on top of the spike train plots.

To learn trajectories from the spike patterns we used multiple linear regression, which was applied to two different tasks. In the representation task, we estimated model parameters based on all 25 trials and tested on one of them. In the generalization task, training was performed on only 24 trials and testing was done using the remaining trial. Both tasks are sketched in Figure 4B. To compare the anisotropic network with a classical reservoir computing approach, we also implemented a randomly connected network on Loihi and exchanged the anisotropic network in our network architecture with a randomly connected network of equal size. We set the parameters of the random network such that the main statistics of both networks match. The firing rate in both networks is in a range of $0.1-0.2$ spikes per number of neurons (Figure 5A bottom) and the mean Fano factor over all trials is relatively similar with $\overline{F F}_{\text {rand }}=$ $0.80 \pm 0.01$ for the randomly connected network and $\overline{F F}_{\text {aniso }}=$ $0.84 \pm 0.002$ for the anisotropic network.

After all data were recorded, in a first step, we prepared the data for the estimation of the regression model. Due to the slow rise in the firing rate of the network (Figure 3C), the very first time steps contain little information. Therefore, we omitted the first 5 time steps which reduces the length of the data set to 210 per trial. Before the linear regression was applied to the spike data, the spike trains were binned in order to smooth our spiking data. We used a sliding window with a width of 10 time steps, which reduced the length of the data set again from 210 to 200 .

Next we used the binned data of the 200 time steps to estimate the regression parameters. In addition to the spiking data from the neurons, an intercept was added such that the number of parameters equal the number of neurons plus one. The linear regression model was performed on the CPU of the host computer, using the spiking data from the readout provided by Loihi. The two different tasks, the representation task and the generalization task, were performed using the spiking data from the anisotropic network as well as those from the randomly connected network. Furthermore, we estimated output weights based on either the pooling layer neurons (72 neurons) or the excitatory neurons of the reservoir (3,600 neurons), see also Figure 1. The excitatory neuron readout serves as a control and compares the pooling layer approach to a traditional readout.

For the estimation based on all excitatory reservoir neurons, we applied an elastic net regularization (Zou and Hastie, 2005) to avoid overfitting, due to the numerous parameters. This regularization approach for regression models simply combines LASSO and ridge regression. We used the fit_regularized function from the statsmodels package in Python, which applies elastic net as

$$
\hat{\beta}=\underset{\beta}{\operatorname{argmin}}\left[\|y-X \beta\|_{2}^{2}+\alpha\left((1-\lambda)\|\beta\|_{2}^{2}+\lambda\|\beta\|_{1}\right)\right] .
$$

In this variant the parameter $\alpha$ determines the degree of regularization and $\lambda$ balances between LASSO ( $L 1$ regularization) and ridge regression ( $L 2$ regularization).

To better compare the predicted function with the target function, we applied a Savitzky-Golay filter (Savitzky and Golay, 1964) to smooth the predicted function. For this we used the savgol_filter function of the Python package scipy. We chose a window length of 21 and an order of the polynomial of 1 as parameters for smoothing.

We trained our algorithm on 7 different trajectories performing ordinary robotic tasks. The tasks are hide, unhide, move down, move up, pick and place, put on top and take down (see e.g., Wörgötter et al. (2020)). Movement data is given in 3 dimensional Cartesian coordinates, resulting in three outputs or - biologically speaking - in three rate coded output neurons.

\section{RESULTS}

We start by demonstrating that the main principles of the anisotropic network are preserved by the Loihi implementation and then confirm that the Loihi-based anisotropic network admits noise-robust spiking dynamics. Based on these findings, we demonstrate that our architecture can learn complex trajectories under noisy input conditions.

\subsection{Implementing the Computer-Based Anisotropic Network on Loihi}

Due to the different hardware architectures, we first assess the extent to which the Loihi-based implementation of the anisotropic network agrees with the computer-based NEST simulation. Please note that it is not our goal to compare two neural network simulators, but to ensure that the anisotropic network implementation on Loihi preserves the main features. For the sake of comparison, we used the same connectivity structure and input positions for both implementations. The networks were initialized at rest and spike patterns were evoked via a spatially-localized input. Raster plots of evoked spike trains indicate that, although the detailed spiking activity is not identical, the overall spiking pattern is mainly preserved (Figure 3A). Accordingly, the mean firing rate of the network for each implementation evolves similarly over time (Figure 3C).

We confirmed the similarity between both implementations quantitatively, comparing the mean firing rate and firing rate variability over several input and network initializations. Figure 3D shows the distribution of mean firing rates over (1) 15 different input positions for the same network connectivity and over (2) 15 different initializations of the network connectivity with a fixed input position. Across input positions in the same network, firing rates for the Loihi implementation were $\bar{f}_{\text {inp }}^{\mathrm{L}}=$ $0.131 \pm 0.008$ and for the NEST implementation $\bar{f}_{\text {inp }}^{\mathrm{N}}=0.118 \pm$ 0.004. Across network initializations, firing rates were $\bar{f}_{\text {init }}^{\mathrm{L}}=$ $0.120 \pm 0.013$ for Loihi and $\bar{f}_{\text {init }}^{\mathrm{L}}=0.113 \pm 0.009$ for NEST. In 


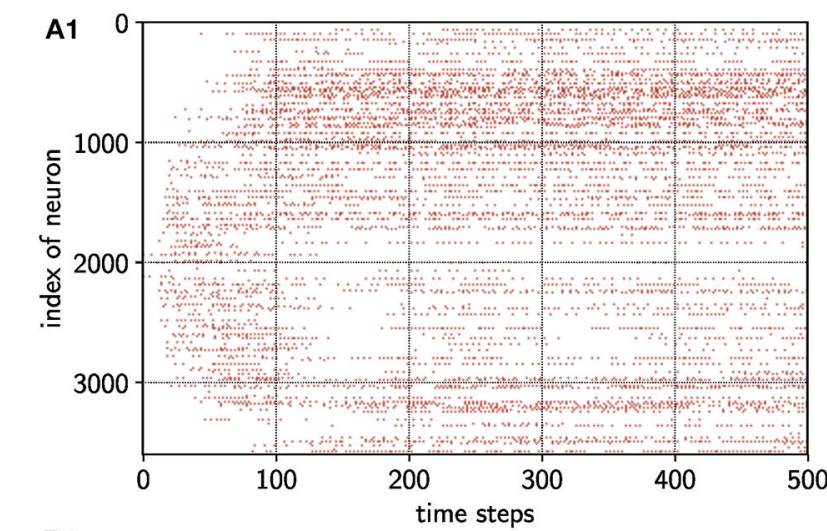

B1

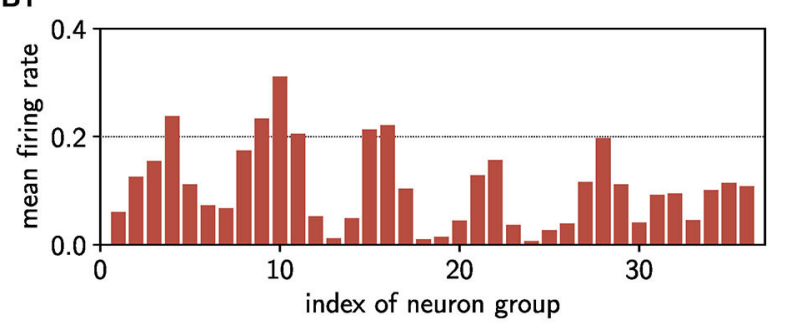

C

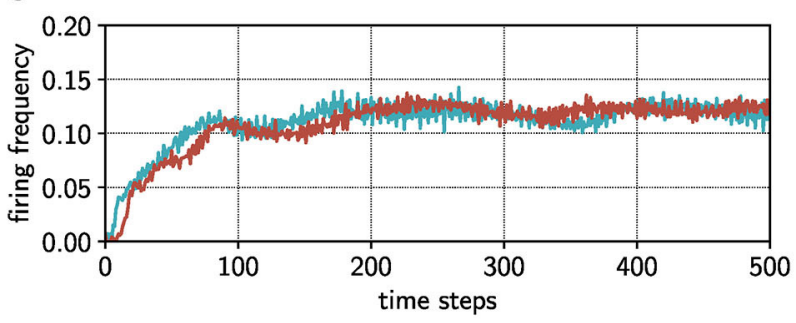

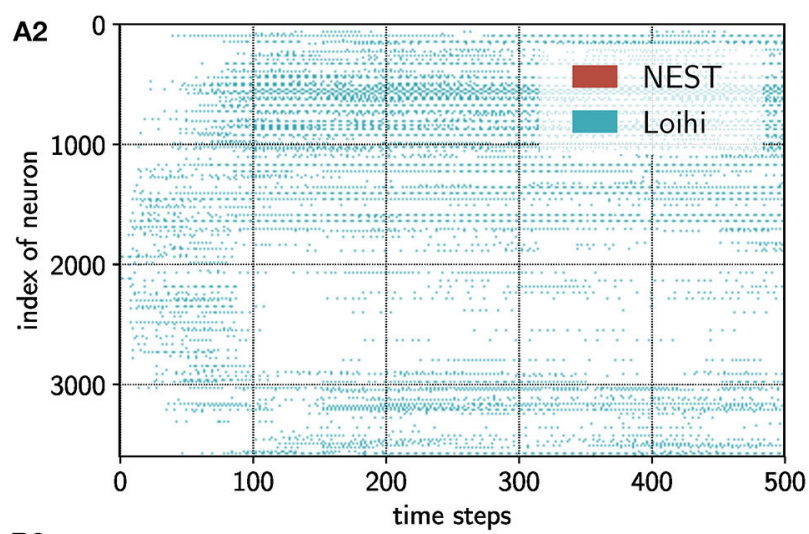

B2

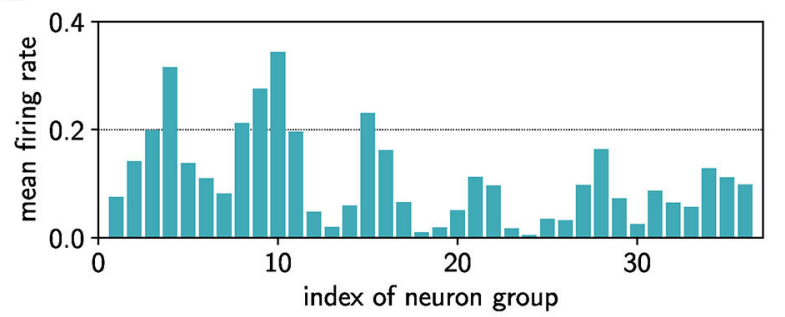

D

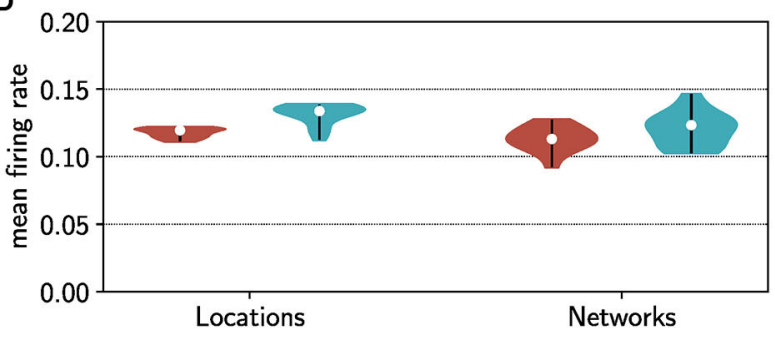

FIGURE 3 | One example of the anisotropic network implementation on Loihi compared to activity statistics of the NEST simulations. (A) The spiking activity is shown as spike raster plots. Red: NEST simulation; blue: Loihi implementation. Both networks are initialized with the same weight matrix and triggered with the same input. (B) By pooling neurons into groups, each consisting of 100 neurons, and binning across the whole simulation time, we obtain the distribution of mean firing rates (\# spikes / \# neurons per group) for the NEST and Loihi implementation. (C) The time course of the network firing rate (\# spikes / \# neurons) from the NEST and Loihi simulations is depicted. (D) Testing one network initialization with 15 different input locations (Locations) and one location but 15 different network initialization (Networks) for the NEST as well as the Loihi implementation. White dots indicate the median of the mean firing rate (\# spikes / \# neurons) of the 15 simulations for each case.

addition, the ranges (minimum to maximum mean firing rate) of the obtained mean firing rates are very tight and overlap largely between both implementations. For the locations, the values for Loihi are in a range of $0.11 \leq f_{\text {inp }}^{\mathrm{L}} \leq 0.14$ and for NEST in an interval of $0.11 \leq f_{\text {inp }}^{\mathrm{N}} \leq 0.12$. In case of the different initializations, we obtained mean firing rates between $0.10 \leq f_{\text {init }}^{\mathrm{L}} \leq 0.15$ for Loihi and $0.09 \leq f_{\text {init }}^{\mathrm{N}} \leq 0.13$ for the NEST implementation. To compare the variability of the firing rate in both implementations, we evaluated the Fano factor (FF): For different input positions, we obtained a mean of $\overline{F F}_{\text {inp }}^{\mathrm{L}}=$ $0.83 \pm 0.03$ for Loihi and $\overline{F F}_{\text {inp }}^{\mathrm{N}}=0.86 \pm 0.01$ for NEST. In the case of the 15 network initializations, the mean FF for Loihi is $\overline{F F}_{\text {init }}^{\mathrm{L}}=0.84 \pm 0.02$ and $\overline{F F}_{\text {init }}^{\mathrm{N}}=0.86 \pm 0.01$ for NEST. All FF values between Loihi and NEST are very close to each other and indicate that spiking is less variable than a Poisson process.
Given that the neural activity in the anisotropic network forms spatially-localized bumps moving through the network, we next measured its average spatial distribution. For the spike rasters shown in Figure 3A, we pooled the neurons into groups of 100, taking into account the topology of the network (see Figure 1B), and calculated the mean firing frequencies averaged across the whole simulation time. This procedure provides a distribution of the mean activity across the network for both implementations (Figure 3B). Normalizing these distributions and comparing them with a Kolmogorov-Smirnov test reveals that the activity distributions from the NEST- and Loihi-based implementations do not differ significantly $(D=0.11, p=$ $0.97>0.05)$. Hence the spatial structure of activity patterns is similar in both implementations.

Taken together, we conclude that the Loihi implementation matches the NEST-based anisotropic network implementation according to diverse statistics of the network activity. This 


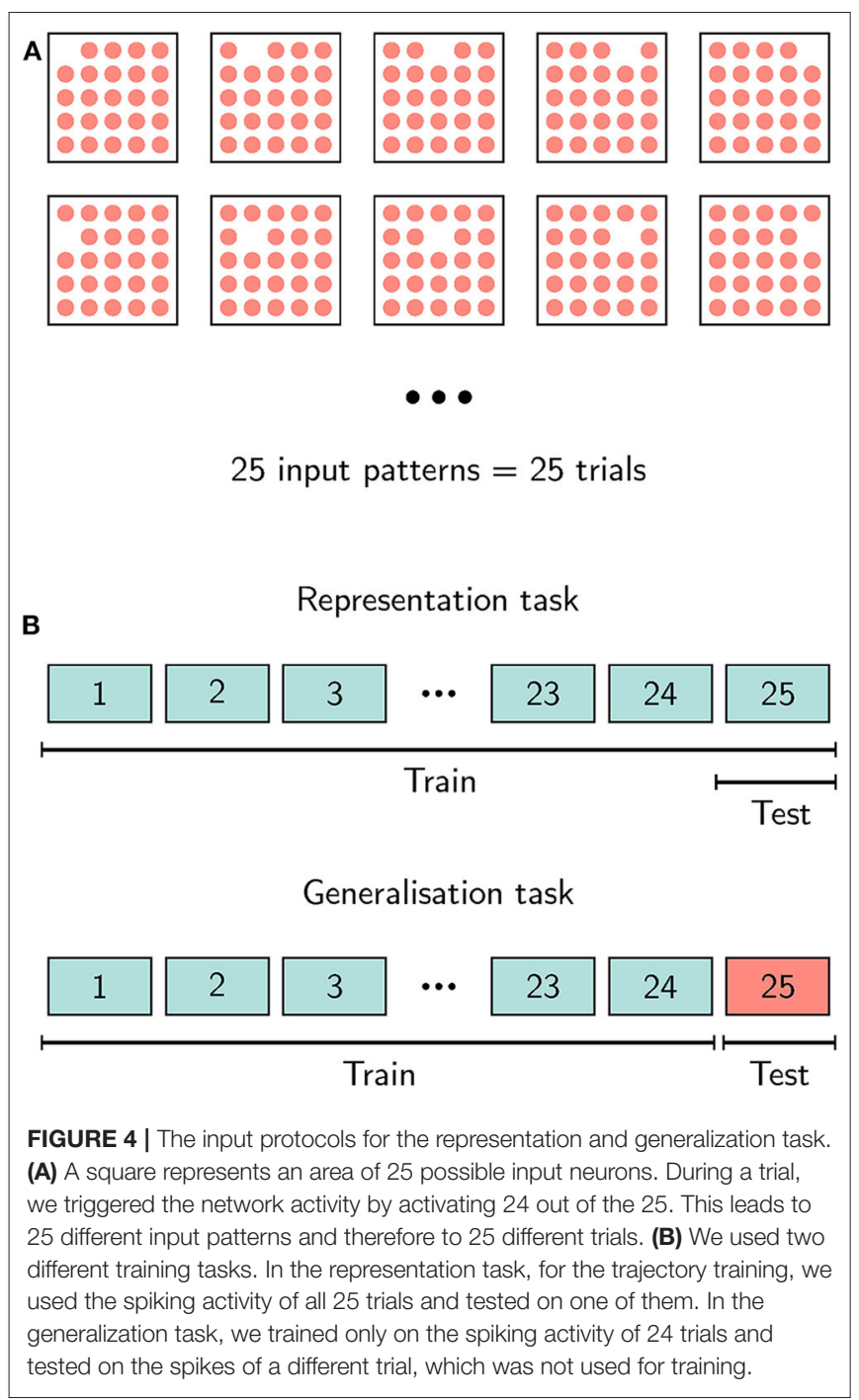

indicates a successful transfer of the core principles of the anisotropic network to neuromorphic hardware despite the differences in architecture.

\subsection{The Loihi Implementation of the Anisotropic Network Is Robust to Input Noise}

Next, to assess the robustness of the Loihi-based anisotropic network to input noise, we evaluate the stability of spiking dynamics. An input pulse is administered to an area of the anisotropic excitatory layer consisting of 25 neurons (Figure 1A). In each trial, 24 of these neurons were activated and a different neuron was systematically excluded from the input, leading to 25 different possible input configurations and, thus, to 25 unique trials (Figure 4).

For each trial the network activity was started with a short input pulse of one time step. We then recorded 200 time steps of activity, stopped the activity manually and activated it again by the next input. The protocol is also indicated on top of Figure 5A.

As a control, we applied the same protocol to a randomly connected network implemented on Loihi and compared it with the anisotropic network implementation. For this, we implemented the same algorithmic architecture, but exchanged the anisotropic network with a randomly connected network of equal size. The spiking activity of the first three trials is shown in Figure 5A1 for the anisotropic network (green) and Figure 5A2 for the randomly connected network (brown). Due to the inhomogeneous connectivity structure, the activity of the anisotropic network spreads out like a stream in the network. Note that, given the torus network topology (see section 2), the activity stream wraps around from neurons with low indices to neurons with high indices (Figure 5A). As expected, in the randomly connected network such a stream-like spread of activity does not form.

The population firing rates progress differently in the anisotropic and randomly connected networks. The mean firing rate of the anisotropic network increases slowly until it reaches a relatively constant rate slightly above 0.1 . The randomly connected network was tuned such that it generates a similar mean population firing rate (see section 2). However, unlike in the anisotropic network, the firing rate does not rise gradually, but instead starts at about $0.1-0.2$ straight away. The slow start in the anisotropic network is due to the relatively small input area and the local connectivity of the network. While moving forward in the 2D-topology, the area of activity grows step by step, which can intuitively be understood as a snowball effect.

In order to measure the stability of the spiking dynamics between different input trials, we calculated the pairwise differences between the spike patterns of all combinations of the 25 trials. The mean and standard deviations of these differences are shown in Figure 5B for both the anisotropic network (green) and the randomly connected network (brown). The differences between trials are much higher over the whole time course for the randomly connected network than for the anisotropic network. For the anisotropic network, the deviations of the trialto-trial differences are very small in the beginning and drift apart over time. To quantify this, we performed a Levene test with three samples at time steps 10,100 , and 190 which revealed that the variance stays constant between the differences of the randomly connected network trials $(W=0.60, p=0.54>$ $0.05)$ but increases for the anisotropic network trials over time $\left(W=208.87, p=3.36 \cdot 10^{-75}<0.05\right)$. This means that, over time, spiking patterns between some trials stay very similar whereas some trial comparisons tend to differ more. Therefore, the anisotropic network tends to slowly diverge with time, which can also be seen by the increasing mean differences. Importantly the mean differences in the anisotropic network remain much lower than the spiking differences between the trials in the randomly connected network, even at the end of the 200 time steps. This clearly demonstrates the stabilizing feature of the anisotropic network.

To visualize differences between the single trials, we reduced the dimensionality of the spiking data by applying principal component analysis (PCA) to all trials (see section 2). The 

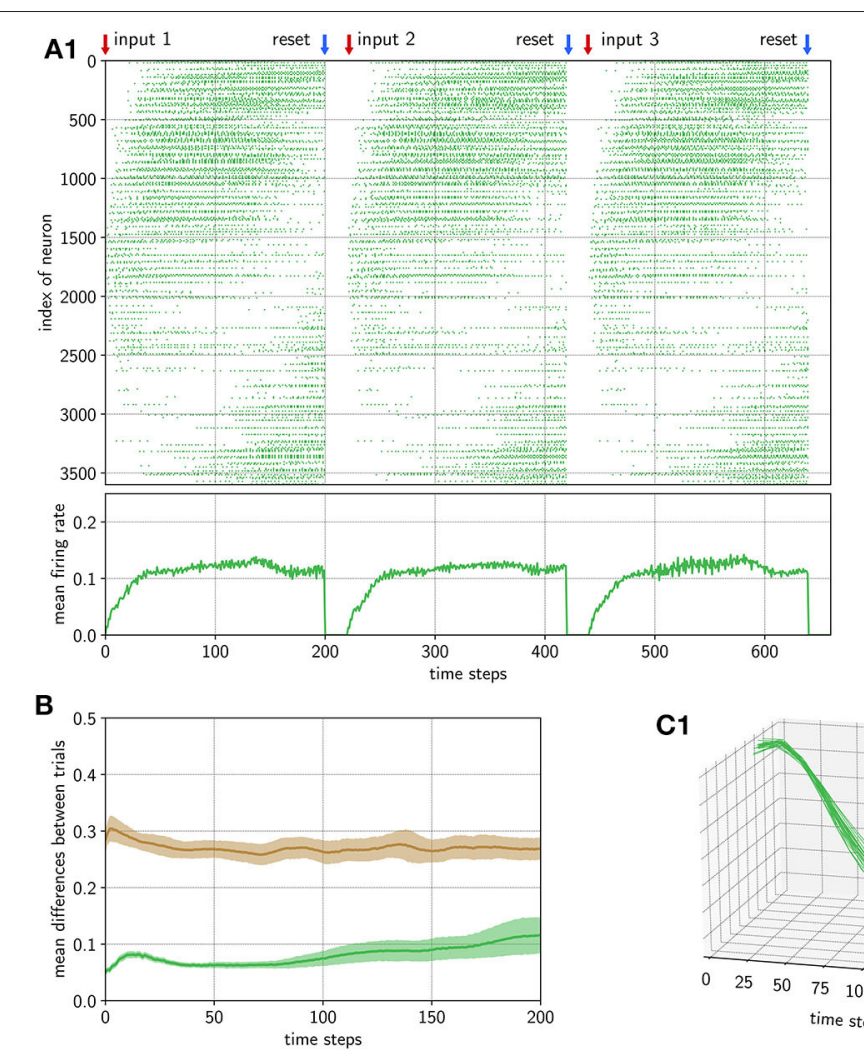

C1
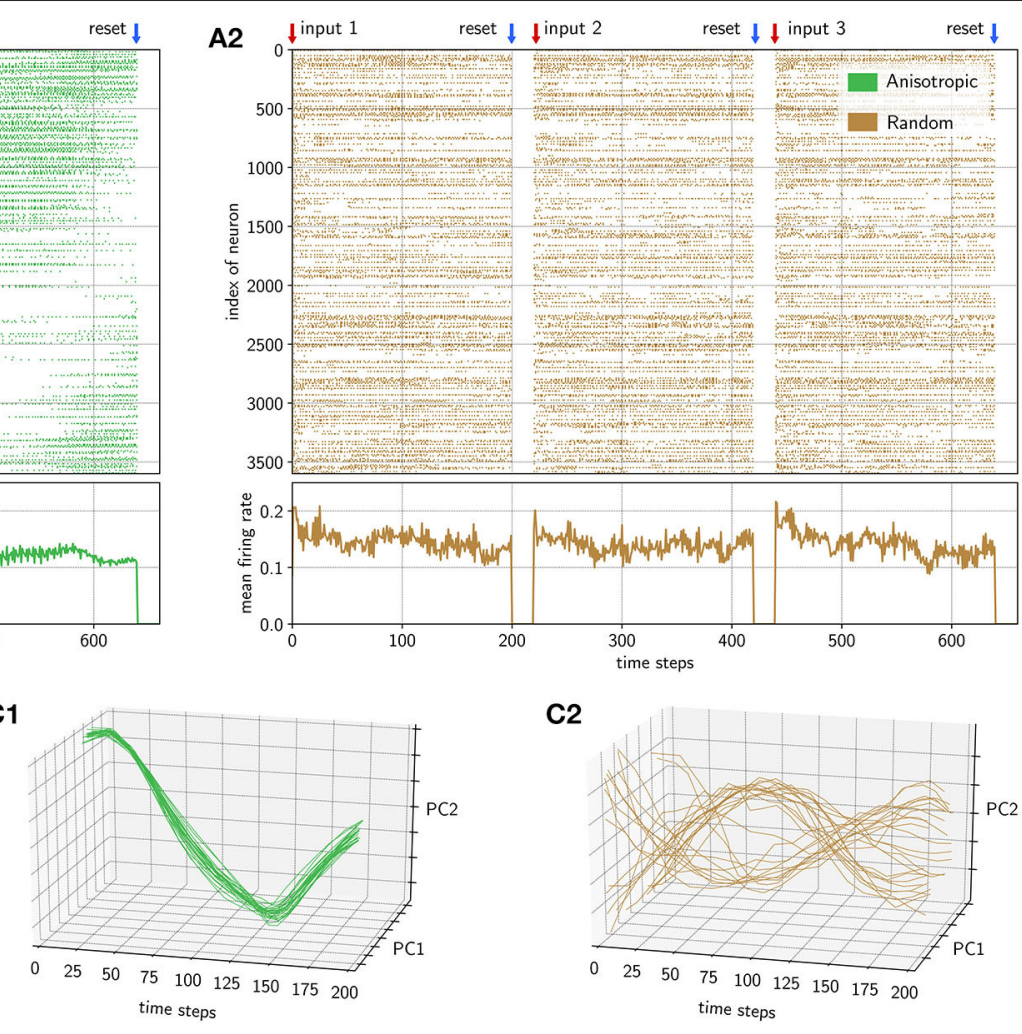

C2

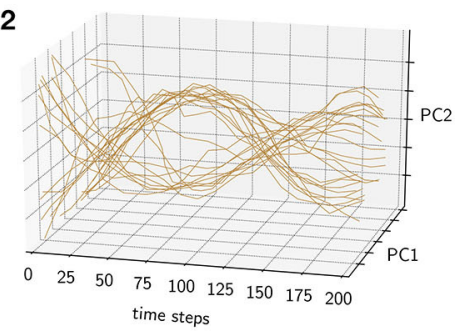

FIGURE 5 | The Loihi implementation of the anisotropic network is robust to varying input conditions, while a randomly connected network is not. (A) Three examplary trials out of the 25 trials are shown for a simulation based on an anisotropic connectivity structure (green) and with randomly initialized weights (brown). The anisotropic structure clearly has a stream-like spiking pattern, where the firing rate starts slowly until it reaches a constant rate. The randomly connected network shows a Poisson-like spiking pattern, where the firing rate starts directly at a high level. (B) The solid lines show the mean difference between all trial combinations and the shaded area indicates the standard deviation of the trial-to-trial differences for the anisotropic (green) and random (brown) network. (C) In the reduced space of the first two principal components of the activity of all 25 trials over time for both networks, we can clearly see that the spiking pattern of the anisotropic network are very similar between trials, while the activity in the randomly connected network differ much more between trials.

results are shown in Figure 5C. For the anisotropic network (Figure 5C1), all trajectories are very similar whereas for the randomly connected network (Figure 5C2) the trajectories differ considerably. We quantified this by calculating statistics in the first dimension of the PCA space. First, we obtained the pairwise normalized mean squared error between all trials for each network type. The normalized mean error between the trials of the randomly connected network is $\mathrm{MSE}_{\text {rand }}=1.66$, while the anisotropic network has a mean error of only $\mathrm{MSE}_{\text {aniso }}=0.03$, which is significantly lower (Mann-Whitney $U$-test: $U=3572.0$, $\left.p=4.27 \cdot 10^{-85}<0.05\right)$. Even though some trajectories seem to follow a common path, in the random network, the mean standard deviation for the first principle component $\bar{\sigma}_{\text {rand }}=$ 2.50 is significantly higher than in the anisotropic network with $\bar{\sigma}_{\text {aniso }}=0.41$ (Mann-Whitney $U$-test: $U=0.0, p=1.56 \cdot 10^{-8}<$ $0.05)$. This indicates sufficient stability over 200 time steps for the anisotropic network.

Taken together, this shows the ability of the anisotropic network to produce stable spiking dynamics under noisy input conditions. In addition it confirms the successful implementation of the network on Loihi. In the next step we will use this intrinsic stability feature of the anisotropic network to learn robust trajectories and examine if our network produces sufficient variability to learn arbitrary functions.

\subsection{Learning Robust Trajectories}

After having tested and demonstrated the stabilizing feature of the anisotropic network, we aimed to use its robustness to train arbitrary output trajectories. This step makes use of the underlying network architecture shown in Figure $\mathbf{1}$ and adds a linear regression model on top of this architecture for a robot control task. The overall algorithm contains the initialization, creation and simulation of the anisotropic network, which is running on the neuromorphic hardware Loihi, and the output learning of the trajectories, which is calculated on the host CPU.

To show the robustness of this algorithm, we learned 7 different 3D-trajectories commonly used in robotic research, like pick-and-place or put-on-top (see section 2). Using these target functions, we applied two different tasks, a representation and a generalization task, as shown in Figure 4B. In the representation task we estimated the linear regression model based on all 25 trials and predicted one of them, showing that 
the variability in the anisotropic network is sufficient to learn an arbitrary function. To show the ability of our algorithm to robustly generalize for variations in the input, we also apply a generalization task, where we estimate the regression model on 24 trials and predicted the trajectory for an unseen trial.

As before, here we also compare the performance of the anisotropic network with the randomly connected network as a control. For our algorithm we estimate our model based on the 72 pooling layer neurons, which we can read out efficiently from the chip. Since we reduce the parameter space of the linear regression model by using only the spiking activity of the pooling layer neurons as data, we also estimated all models based on the 3, 600 excitatory reservoir neurons for comparison.

Results for the representation task, based on all excitatory neurons, revealed that the excitatory reservoir neurons contain enough variability to represent an arbitrary output function with high accuracy. An example is shown in Supplementary Figure 1A1 for the randomly connected network and in Supplementary Figure 1A2 for the anisotropic network. If the model was estimated on the 72 pooling layer neurons the number of available parameters is heavily decreased by a factor of 50 . But still the amount of information seems to be satisfactory for the anisotropic network (Supplementary Figure 1A4), but not for the randomly connected case (Supplementary Figure 1A3). Normalized root mean squared error between the predicted trajectory and the target trajectory, averaged over 7 3D-trajectories, are shown in Figure 6A1. The errors for all trajectories using the spiking activity of the 3,600 excitatory reservoir neurons are very low (left plot) for both networks, but interestingly even lower for the anisotropic network. For the errors of the estimation based on the pooling layer neurons (right plot in Figure 6A1), the mean error over all trajectories is still low for the anisotropic network $e_{\text {aniso }}=0.02 \pm 0.003$, compared to the randomly connected network $e_{\text {rand }}=0.33 \pm 0.07$. Due to the inhomogeneous weight structure and the streamlike spread of spiking activity in the anisotropic network, the neurons in the pooling layer can maintain variability, as can be seen in Supplementary Figure 1B1. Intuitively, since nearby neurons have correlated activity patterns, pooling over them preserves information. In contrast, as shown in Supplementary Figure 2B2, the spiking activity in the pooling layer of the randomly connected network simply produces downsampled random spiking activity and therefore reduced variability.

In the generalization task, the parameters for the movement trajectory were estimated based on the spiking activity of 24 trials. We then predicted the same trajectory based on the spiking activity elicited by a 25 th trial, not seen during training. This task was designed to test the robustness of the system to a variation in initial conditions. To compare the classical reservoir computing approach with our network architecture, we trained the network based on all 3,600 neurons and on the 72 output neurons. In addition, this tested the ability of the pooling neurons to preserve sufficient variability while reducing the number of parameters.

For the full network read-out, we applied a linear regression model based on all excitatory neurons of the anisotropic network.
Since fitting a model based on all 3,600 neurons requires many parameters, here we used an elastic net regularization estimation method (see section 2) to reduce the number of parameters and to avoid overfitting. Optimizing the regularization parameters resulted in $\alpha=0.001$ and $\lambda=0.05$. For the pooling layer readout, we estimated a linear regression model based on the pooling layer neurons without regularization.

In Figure 6A2, we show the average normalized root-meansquared deviation over 7 trajectories. In both cases (using excitatory neurons or pooling layer neurons) the error of the anisotropic network is much lower, showing that the anisotropic network has a better performance compared to a classical randomly connected network.

For the network architecture with the randomly connected network, the elastic net approach, based on all excitatory neurons, has a better performance than the linear regression approach, based on the pool neurons $(t$-test: $t=-4.24, p=0.0001<$ $0.05)$. Interestingly, the error for the anisotropic network is lower when the trajectories are estimated based on the pool neurons compared to the excitatory neurons (Mann-Whitney $U$-test: $\left.U=47.0, p=6.75 \cdot 10^{-6}<0.05\right)$. This indicates that, for the anisotropic network, the pooling layer is an equivalent, or even better regularization method compared to the elastic net approach with all excitatory neurons.

Figure 6B shows all three dimensions of the predicted trajectory over time for a hide movement. The overall trajectory is shown in Figure 6C. We also calculated a smoothed version, using a Savitzky-Golay filter (Savitzky and Golay, 1964) (see section 2), to better compare the prediction with the target. This shows that the anisotropic network implemented on Loihi, combined with the pooling layer, contains sufficient variability to represent complex 3D trajectories while at the same time remaining stable for at least 200 time steps.

\subsection{Simulation on Loihi in Real-Time}

In addition to evaluating the stability of the system, we also looked at the speed of the network simulation. The data we used came from a Kuka robot arm, which can run fluently with an output frequency of $100 \mathrm{~Hz}$, therefore the 200 time steps equal $2 \mathrm{~s}$ of movement. In the following we denote this reference as "real-time." To achieve a real-time output of spiking data from the Loihi chip, the speed of the simulation of the neurons and the data transfer from the chip to the host must be higher than the necessary data frequency of the robot for a smooth movement.

The simulation of these 200 time steps requires $t_{3,600}^{\text {aniso }}=$ $15.73 s$ for one trial on Loihi, when all 3,600 excitatory reservoir neurons were read out from the system. This speed is about 8 times slower than real-time. When reading out only from the 72 pooling neurons, the speed increases to $t_{72}^{\text {aniso }}=1.49 \mathrm{~s}$ per trial, which is $25 \%$ faster than real-time and therefore well-suited for robot control.

The simulation speed of the anisotropic network $\left(t_{3,600}^{\text {aniso }}=\right.$ $15.73 s \& t_{72}^{\text {aniso }}=1.49 s$ per trial) and the randomly connected network $\left(t_{3,600}^{\text {rand }}=16.11 s \& t_{72}^{\text {rand }}=1.73\right.$ per trial $)$ were nearly the same, which is expected since the number of neurons is the same and the number of synapses is similar. Thus, the anisotropic 


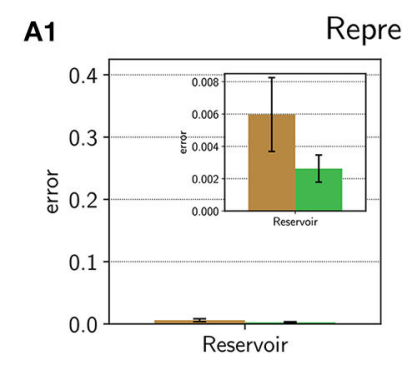

\section{B}
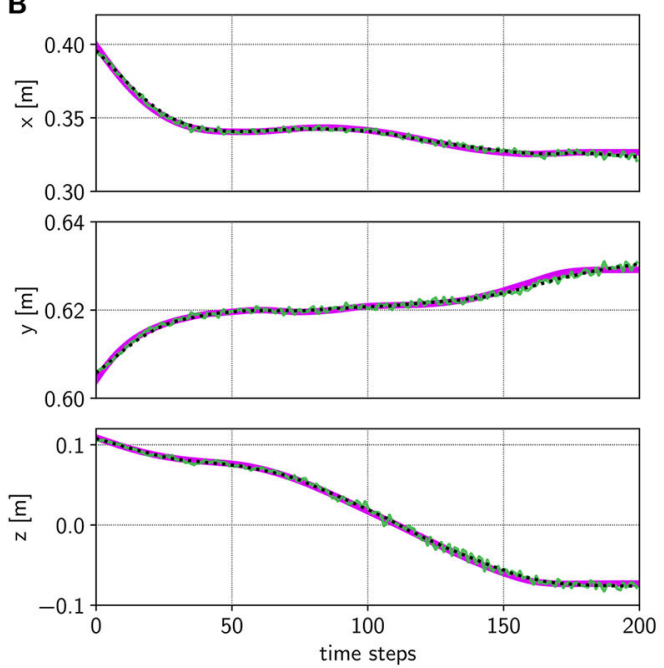

A2

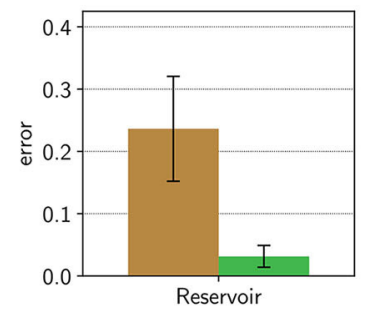

Generalisation

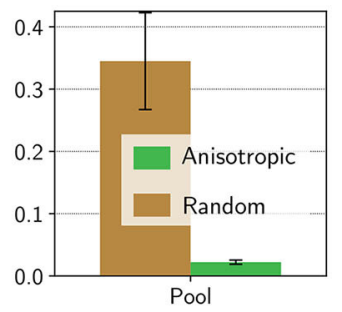

C

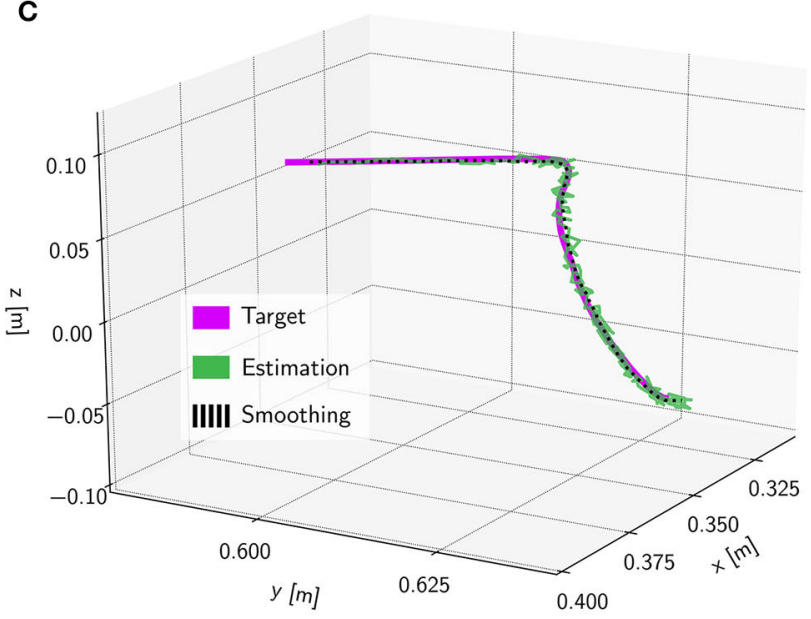

FIGURE 6 | Predicting a trajectory on the spiking activity. (A) We predicted 7 different trajectories with 3 dimensions each and calculated the error regarding the target trajectory using a normalized root-mean-square error. In all cases, the anisotropic network has a lower error than the randomly connected network. (B) For the hide movement, we show one example of the predicted trajectory of the anisotropic network for each of the 3 dimensions. Here, we used the pooling neurons (green) in comparison with the target (pink) and a smoothed variant of the prediction (black dotted). The whole trajectory is shown in (C).

network has, in terms of speed, no disadvantage compared to the randomly connected network.

Therefore, the pooling layer does not only reduce the sensitivity of the system but also helps to speed up the system considerably. Together, this supports robotic applications where trajectories can be stored and replayed robustly in real-time.

\section{DISCUSSION}

We aimed to develop an algorithm for neuromorphic hardware, which provides stable spiking dynamics under noisy input conditions, in order to make use of the low power neuromorphic chips for future autonomous systems. For this, we derived an algorithm to store and control robotic movement sequences that unfold on a control-relevant timescale of seconds. To validate our approach, we chose a set of 2-s-long robot arm movements that were triggered by noisy inputs.

For our approach we chose a recently developed spiking neural network (Spreizer et al., 2019) with an inhomogeneous weight structure. In a first step, we successfully transferred the main principles of this network to the Loihi research chip from Intel (Davies et al., 2018), a neuromorphic hardware architecture implementing spiking neurons. In a second step, we tested the stability of the anisotropic network implementation and compared its stability to a classical randomly connected network, similar to echo state networks (Jaeger, 2001, 2007) or liquid state machines (Maass et al., 2002). We finally used a pooling layer (Figure 1) to efficiently read out spiking data from the chip. Using these spiking data we were able to learn $3 \mathrm{D}$ trajectories in a noiserobust way (Figure 6C). The pooling layer successfully increased the simulation speed to faster than real-time. It was also intended to make the spiking activity more invariant to small changes in the network, which is the exact purpose of using pooling layers in deep neural networks (Goodfellow et al., 2016, Chapter 9.3; Boureau et al., 2010). A pooling layer has been applied to spiking neural networks before (Tavanaei and Maida, 2017; Tavanaei et al., 2019), but - to the best of our knowledge - such a structure has never been applied to enhance the performance of read-outs from recurrent network architectures. The fact that the pooling layer improved performance for the anisotropic network in our study indicates that implementing pooling layers in reservoir computing architectures could be useful in other cases, for example when the reservoir has spatially-dependent connectivity (Maass et al., 2002), and especially for reducing parameters on algorithms running on neuromorphic hardware.

Taken together, in this study we provide an algorithm for storing stable trajectories in spiking neural networks, optimized 
for the neuromorphic hardware Loihi. The network architecture is capable of executing these trajectories on demand in realtime given noisy, and even never-before-seen, inputs. While an exhaustive exploration of the parameter space remains the subject of future work, we have shown that the anisotropic network admits stable sequences with sufficient variability for output learning across hundreds of milliseconds, making it suitable for applications reaching far beyond motor control. Further, we demonstrated that spike-based pooling can implement on-chip regularization for the ansiotropic network, improving read out speed and accuracy. In contrast, in the randomly connected network nearby neurons show uncorrelated activity and spatial pooling has no benefit. Thus, spatial pooling in locally-connected SNNs proved to be a promising feature, specifically for realtime robotic control on neuromorphic hardware. Importantly, we provide the first neuromorphic implementation which has no global learning or adaptation mechanism and produces noiserobust spiking patterns on a control-relevant timescale with sufficient variability to learn arbitrary functions.

While other approaches employing spiking neural networks exist, in general they fail to meet at least one of the mentioned criteria. This means, in their current form, these models are either not implementable on neuromorphic hardware or do not produce sequences that are stable, variable and long enough. We briefly describe these models and highlight how they may be adapted for neuromorphic implementation.

Laje and Buonomano (2013) presented an "innate training" approach. The network was initialized with a short input pulse and a modified FORCE algorithm (Sussillo and Abbott, 2009) was used to train the recurrent connections. This stabilizes the innate structure of the recurrent connections and allows a network state between chaotic and locally stable activity patterns. A trained output trajectory was robust to perturbations, due to the tuned recurrent weights. Unfortunately this algorithm uses a rate coded network and non-local learning rules, both of which are not applicable for most neuromorphic systems.

Pehlevan et al. (2018) analyzed different approaches to solve the stability-variability trade-off in the context of songbird songs. One additional and important criterion for their evaluation was the ability of an algorithm to provide temporal flexibility, such that outputs can be replayed faster or slower. They concluded that a synfire chain model fits best to solve this task. While this approach seems to model the dynamics underlying songbird songs with flexible timing, synfire chains have a feedforward structure which makes them less flexible than recurrent network types.

Hennequin et al. (2014) put more focus on getting stable output from unstable initial conditions. They used an optimization algorithm to build an inhibitory structure that helps to stabilize the excitatory activity. More precisely, the strength of existing inhibitory connections was changed or new inhibitory synapses were created or removed using an algorithm based on a relaxation of the spectral abscissa of the weight matrix (Vanbiervliet et al., 2009). With this they obtained relatively stable spiking dynamics. Interestingly, this approach is similar to our study in a sense that both approaches focus on the weight matrix. While their proposed solution to the stability-variability trade-off is promising, so far the algorithm has mainly been tested with rate coded networks. A more elaborate analysis with a spiking neural network would be of interest.

Another recent approach involves multiplexing oscillations in a spiking neural network (Miall, 1989; Vincent-Lamarre et al., 2020). Two input units inject sine-waves into a reservoir of neurons and the spiking dynamics in the reservoir follow a stable and unique pattern, which enables the learning of a long and stable output. Compared to our algorithm, the oscillating units provide a continuous input to the network. We see this approach as a potential alternative to the anisotropic network for robotic control. Interestingly, stability is encoded in time rather than space, which raises the question whether this approach could be combined with a pooling layer, reflecting temporal structure instead of spatial structure.

Maes et al. (2020) trained a recurrently connected spiking network such that small groups of neurons become active in succession and thus provide the basis for a simple index code. Via a supervisor signal, output neurons are trained to become responsive to a particular group or index from the recurrent network and, thus, fire in a temporal order encoded in the feed-forward weights to the output layer. Importantly, learning within the recurrent network and from the recurrent network to the output layer is done using spike-timing dependent plasticity. However, as is, their implementation has a few small, but likely reconcilable, incompatibilities with the neuromorphic hardware considered here. For example, learning and synaptic normalization is only local to the neuron, and not to the synapse and they rely on adaptive exponential integrate and fire neurons, which are not implemented by Loihi. With some modifications, their model may provide another neuromorphically implementable approach.

While our approach provides an algorithm for storing stable trajectories, our two-chip Loihi system is limited in the number of neurons available, constrained mainly by the high number of synapses in our recurrent network. Since this limitation is mainly caused by the current NxSDK software and not by hardware, we expect an improvement in upcoming releases. With more neurons available we expect even better stability, reducing the last remaining variations in our predictions and allowing even longer movement actions, beyond $2 \mathrm{~s}$. At this point, further investigation of how performance depends on network size, network parameters, and pooling layer configuration will be of interest.

With more neurons available, one could add multiple inputs to the network. We hypothesize that nearby input locations lead to similar activity patterns, while input regions far from each other produce distinct activity patterns. This behavior could be used to train multiple trajectories from different input locations. With this, more complex robotic control tasks could be performed, beyond the generation of single trajectories.

One general hurdle in developing neuromorphic implementations is the difficulty in transferring existing spiking neural network models from CPU-based implementations to neuromorphic hardware. As outlined in the section 2, Loihi provides a fixed hardware-implemented neuron model. It is possible to adjust parameters, but not the neuron model itself. Therefore, a perfect match between traditional simulators like NEST (Gewaltig and Diesmann, 2007) or Brian2 (Stimberg 
et al., 2019) and neuromorphic hardware, like Loihi, is in general an issue for future neuromorphic algorithms. Efficient methods for translating neuroscientific models to Loihi is the subject of current work.

Finally, to complete the algorithm for autonomous use cases, in which Loihi is able to control a robot independently, an on-chip output learning algorithm is vital. This requires the implementation of an output neuron on the chip, with appropriate on-chip output weights. It is already possible to train weights offline and transfer them to Loihi, applied for example in Nengo Loihi (Bekolay et al., 2014; Hampo et al., 2020). We expect that the on-chip regularization inherent in spatial pooling will improve the robustness of future online output learning algorithms.

\section{CONCLUSION}

Taken together, we developed an algorithm which can serve as a basic unit in robotic applications. The anisotropic network structure offers stability against noisy inputs and the overall architecture, especially using the pooling layer, paves the way for further steps in the development of algorithms for neuromorphic hardware. Our study proposes an algorithm based on intrinsic self-stabilizing features of a well-initialized anisotropic connectivity structure, which can overcome the instability problem of spiking neural networks and support robust outputs on a timescale of seconds.

\section{DATA AVAILABILITY STATEMENT}

The PeleNet framework for Loihi, which was written for this study, can be found on GitHub (https://github.com/sagacitysite/

\section{REFERENCES}

Amit, D. J. (1992). Modeling Brain Function: The World of Attractor Neural Networks. Cambridge: Cambridge University Press.

Bekolay, T., Bergstra, J., Hunsberger, E., DeWolf, T., Stewart, T., Rasmussen, D., et al. (2014). Nengo: a Python tool for building large-scale functional brain models. Front. Neuroinform. 7, 1-13. doi: 10.3389/fninf.2013. 00048

Blouw, P., Choo, X., Hunsberger, E., and Eliasmith, C. (2019). "Benchmarking keyword spotting efficiency on neuromorphic hardware," in Proceedings of the 7th Annual Neuro-inspired Computational Elements Workshop (New York, NY; Albany, NY: Association for Computing Machinery), 1:8. doi: 10.1145/3320288.3320304

Boureau, Y.-L., Ponce, J., and LeCun, Y. (2010). "A theoretical analysis of feature pooling in visual recognition," in Proceedings of the 27th International Conference on Machine Learning (ICML-10) (Madison, WI: Omnipress), 111-118.

Brunel, N. (2000). Dynamics of networks of randomly connected excitatory and inhibitory spiking neurons. J. Physiol. 94, 445-463. doi: 10.1016/S0928-4257(00)01084-6

Davies, M., Srinivasa, N., Lin, T.-H., Chinya, G., Cao, Y., Choday, S. H., et al. (2018). Loihi: a neuromorphic manycore processor with on-chip learning. IEEE Micro 38, 82-99. doi: 10.1109/MM.2018.112130359

DeWolf, T., Stewart, T. C., Slotine, J.-J., and Eliasmith, C. (2016). A spiking neural model of adaptive arm control. Proc. R. Soc. B Biol. Sci. 283:20162134. doi: $10.1098 /$ rspb. 2016.2134 pelenet/tree/neurorobotics). The data that support the findings of this study are available from the corresponding author on request.

\section{AUTHOR CONTRIBUTIONS}

AL contributed the network simulations. CM contributed the Loihi implementation, including the Pelenet framework. CT acquired funding and supervised the study. All authors designed the study and reviewed the manuscript.

\section{FUNDING}

The research was funded by the H2020-FETPROACT project Plan4Act (\#732266) [CM, AL, CT], by the German Research Foundation (\#419866478) [AL, CT], and by the Intel Corporation via a gift without restrictions.

\section{ACKNOWLEDGMENTS}

The authors are thankful to Osman Kaya for providing Kuka robot trajectories, to Arvind Kumar and Lukas Ruff for helpful discussions, and to Tristan Stöber for improving the text. All of them helped to improve the quality of this work. Furthermore, we thank the Intel Corporation for providing access to their Loihi chip.

\section{SUPPLEMENTARY MATERIAL}

The Supplementary Material for this article can be found online at: https://www.frontiersin.org/articles/10.3389/fnbot. 2020.589532/full\#supplementary-material

Eliasmith, C., and Anderson, C. H. (2004). Neural Engineering: Computation, Representation, and Dynamics in Neurobiological Systems. Cambridge, MA: MIT Press.

Furber, S. B., Galluppi, F., Temple, S., and Plana, L. A. (2014). The SpiNNaker project. Proc. IEEE 102, 652-665. doi: 10.1109/JPROC.2014.2304638

Gewaltig, M.-O., and Diesmann, M. (2007). Nest (neural simulation tool). Scholarpedia 2:1430. doi: 10.4249/scholarpedia.1430

Goodfellow, I., Bengio, Y., and Courville, A. (2016). Deep Learning. Cambridge, MA: MIT Press, 330-334.

Hampo, M., Fan, D., Jenkins, T., DeMange, A., Westberg, S., Bihl, T., et al. (2020). "Associative memory in spiking neural network form implemented on neuromorphic hardware," in International Conference on Neuromorphic Systems 2020, 1-8. doi: 10.1145/3407197.3407602

Hennequin, G., Vogels, T. P., and Gerstner, W. (2014). Optimal control of transient dynamics in balanced networks supports generation of complex movements. Neuron 82, 1394-1406. doi: 10.1016/j.neuron.2014.04.045

Hutt, A. (2008). Local excitation-lateral inhibition interaction yields oscillatory instabilities in nonlocally interacting systems involving finite propagation delay. Phys. Lett. A 372, 541-546. doi: 10.1016/j.physleta.2007.08.018

Ijspeert, A. J., Nakanishi, J., and Schaal, S. (2002). "Movement imitation with nonlinear dynamical systems in humanoid robots," in Proceedings 2002 IEEE International Conference on Robotics and Automation (Cat. No. 02CH37292), Vol. 2 (Washington, DC: IEEE), 1398-1403. doi: 10.1109/ROBOT.2002.1014739

Itskov, V., Curto, C., Pastalkova, E., and Buzsáki, G. (2011). Cell assembly sequences arising from spike threshold adaptation keep 
track of time in the hippocampus. J. Neurosci. 31, 2828-2834. doi: 10.1523/JNEUROSCI.3773-10.2011

Jaeger, H. (2001). The "Echo State" Approach to Analysing and Training Recurrent Neural Networks-With an Erratum Note. German National Research Center for Information Technology, Bonn. GMD Technical Report.

Jaeger, H. (2007). Echo state network. Scholarpedia 2:2330. doi: 10.4249/scholarpedia.2330

Khalastchi, E., Kaminka, G. A., Kalech, M., and Lin, R. (2011). "Online anomaly detection in unmanned vehicles," in The 10th International Conference on Autonomous Agents and Multiagent Systems (Richland, SC; Taipei: International Foundation for Autonomous Agents and Multiagent Systems), $115-122$.

Laje, R., and Buonomano, D. V. (2013). Robust timing and motor patterns by taming chaos in recurrent neural networks. Nat. Neurosci. 16:925. doi: $10.1038 / \mathrm{nn} .3405$

Lee, C., Panda, P., Srinivasan, G., and Roy, K. (2018). Training deep spiking convolutional neural networks with stdp-based unsupervised pre-training followed by supervised fine-tuning. Front. Neurosci. 12:435. doi: $10.3389 /$ fnins.2018.00435

Lin, C.-K., Wild, A., Chinya, G. N., Cao, Y., Davies, M., Lavery, D. M., et al. (2018). Programming spiking neural networks on intel's Loihi. Computer 51, 52-61. doi: 10.1109/MC.2018.157113521

London, M., Roth, A., Beeren, L., Häusser, M., and Latham, P. E. (2010). Sensitivity to perturbations in vivo implies high noise and suggests rate coding in cortex. Nature 466, 123-127. doi: 10.1038/nature09086

Maass, W. (1997). Networks of spiking neurons: the third generation of neural network models. Neural Netw. 10, 1659-1671. doi: $10.1016 / 50893-6080(97) 00011-7$

Maass, W., Natschläger, T., and Markram, H. (2002). Real-time computing without stable states: a new framework for neural computation based on perturbations. Neural Comput. 14, 2531-2560. doi: 10.1162/089976602760407955

Maes, A., Barahona, M., and Clopath, C. (2020). Learning spatiotemporal signals using a recurrent spiking network that discretizes time. PLoS Comput. Biol. 16:e1007606. doi: 10.1371/journal.pcbi.1007606

Miall, C. (1989). The storage of time intervals using oscillating neurons. Neural Comput. 1, 359-371. doi: 10.1162/neco.1989.1.3.359

Murray, J. M. et al. (2017). Learning multiple variable-speed sequences in striatum via cortical tutoring. eLife 6:e26084. doi: 10.7554/eLife.26084

Nachstedt, T., and Tetzlaff, C. (2017). Working memory requires a combination of transient and attractor-dominated dynamics to process unreliably timed inputs. Sci. Rep. 7, 1-14. doi: 10.1038/s41598-017-02471-z

Naseer, T., Burgard, W., and Stachniss, C. (2018). Robust visual localization across seasons. IEEE Trans. Robot. 34, 289-302. doi: 10.1109/TRO.2017.2788045

Neckar, A., Fok, S., Benjamin, B. V., Stewart, T. C., Oza, N. N., Voelker, A. R., et al. (2018). Braindrop: a mixed-signal neuromorphic architecture with a dynamical systems-based programming model. Proc. IEEE 107, 144-164. doi: 10.1109/JPROC.2018.2881432

Pehlevan, C., Ali, F., and Ölveczky, B. P. (2018). Flexibility in motor timing constrains the topology and dynamics of pattern generator circuits. Nat. Commun. 9, 1-15. doi: 10.1038/s41467-018-03261-5

Perlin, K. (1985). An image synthesizer. SIGGRAPH Comput. Graph. 19, 287-296. doi: $10.1145 / 325165.325247$

Roxin, A., Brunel, N., and Hansel, D. (2005). Role of delays in shaping spatiotemporal dynamics of neuronal activity in large networks. Phys. Rev. Lett. 94:238103. doi: 10.1103/PhysRevLett.94.238103

Savitzky, A., and Golay, M. J. (1964). Smoothing and differentiation of data by simplified least squares procedures. Anal. Chem. 36, 1627-1639. doi: 10.1021/ac60214a047

Schemmel, J., Briiderle, D., Griibl, A., Hock, M., Meier, K., and Millner, S. (2010). "A wafer-scale neuromorphic hardware system for large-scale neural modelling," in Proceedings of 2010 IEEE International Symposium on Circuits and Systems (Paris: IEEE), 1947-1950. doi: 10.1109/ISCAS.2010.5536970

Sompolinsky, H., Crisanti, A., and Sommers, H.-J. (1988). Chaos in random neural networks. Phys. Rev. Lett. 61:259. doi: 10.1103/PhysRevLett.61.259
Spreizer, S., Aertsen, A., and Kumar, A. (2019). From space to time: spatial inhomogeneities lead to the emergence of spatiotemporal sequences in spiking neuronal networks. PLoS Comput. Biol. 15:e1007432. doi: 10.1371/journal.pcbi.1007432

Spreizer, S., Angelhuber, M., Bahuguna, J., Aertsen, A., and Kumar, A. (2017). Activity dynamics and signal representation in a striatal network model with distance-dependent connectivity. eNeuro 4. doi: 10.1523/ENEURO.0348-16.2017

Stimberg, M., Brette, R., and Goodman, D. F. (2019). Brian 2, an intuitive and efficient neural simulator. eLife 8:e47314. doi: 10.7554/eLife.47314

Sussillo, D., and Abbott, L. F. (2009). Generating coherent patterns of activity from chaotic neural networks. Neuron 63, 544-557. doi: $10.1016 /$ j.neuron.2009.07.018

Tang, G., Shah, A., and Michmizos, K. P. (2019). Spiking neural network on neuromorphic hardware for energy-efficient unidimensional slam. arXiv preprint arXiv:1903.02504. doi: 10.1109/IROS40897.2019. 8967864

Tavanaei, A., Ghodrati, M., Kheradpisheh, S. R., Masquelier, T., and Maida, A. (2019). Deep learning in spiking neural networks. Neural Netw. 111, 47-63. doi: 10.1016/j.neunet.2018.12.002

Tavanaei, A., and Maida, A. (2017). "Bio-inspired multi-layer spiking neural network extracts discriminative features from speech signals," in Neural Information Processing, eds L. Derong, X. Shengli, L. Yuanqing, Z. Dongbin,and E.-A. El-Sayed (Cham: Springer International Publishing), 899908. doi: 10.1007/978-3-319-70136-3_95

Tsodyks, M. (1999). Attractor neural network models of spatial maps in hippocampus. Hippocampus 9, 481-489. doi: 10.1002/(SICI) 1098-1063(1999)9:4<481::AID-HIPO14>3.0.CO;2-S

Van Vreeswijk, C., and Sompolinsky, H. (1996). Chaos in neuronal networks with balanced excitatory and inhibitory activity. Science 274, 1724-1726. doi: $10.1126 /$ science.274.5293.1724

Vanbiervliet, J., Vandereycken, B., Michiels, W., Vandewalle, S., and Diehl, M. (2009). The smoothed spectral abscissa for robust stability optimization. SIAM J. Optimizat. 20, 156-171. doi: 10.1137/070704034

Vincent-Lamarre, P., Calderini, M., and Thivierge, J.-P. (2020). Learning long temporal sequences in spiking networks by multiplexing neural oscillations. Front. Comput. Neurosci. 14:78. doi: 10.3389/fncom.2020.00078

Voelker, A. R., and Eliasmith, C. (2017). Methods for applying the neural engineering framework to neuromorphic hardware. arXiv [Preprint]. arXiv: 1708.08133 .

Wörgötter, F., Ziaeetabar, F., Pfeiffer, S., Kaya, O., Kulvicius, T., and Tamosiunaite, M. (2020). Humans predict action using grammar-like structures. Sci. Rep. 10, 1-11. doi: 10.1038/s41598-020-60923-5

York, L. C., and Van Rossum, M. C. (2009). Recurrent networks with short term synaptic depression. J. Comput. Neurosci. 27:607. doi: 10.1007/s10827-009-0172-4

Zheng, P., and Triesch, J. (2014). Robust development of synfire chains from multiple plasticity mechanisms. Front. Comput. Neurosci. 8:66. doi: 10.3389/fncom.2014.00066

Zou, H., and Hastie, T. (2005). Regularization and variable selection via the elastic net. J. R. Stat. Soc. Ser. B 67, 301-320. doi: 10.1111/j.1467-9868.2005.0 0503.x

Conflict of Interest: The authors declare that the research was conducted in the absence of any commercial or financial relationships that could be construed as a potential conflict of interest.

Copyright (C) 2020 Michaelis, Lehr and Tetzlaff. This is an open-access article distributed under the terms of the Creative Commons Attribution License (CC BY). The use, distribution or reproduction in other forums is permitted, provided the original author(s) and the copyright owner(s) are credited and that the original publication in this journal is cited, in accordance with accepted academic practice. No use, distribution or reproduction is permitted which does not comply with these terms. 